\title{
Modeling of Layered Fuzzy Facial Expression Generation
}

\author{
Yu-Li Xue, Xia Mao, Zheng Li, and Wei-He Diao \\ School of Electronic and Information Engineering, Beijing University of Aeronautics and \\ Astronautics, Beijing 100083, China \\ YuLi_Xue@ee.buaa.edu.cn, moukyou@buaa.edu.cn
}

\begin{abstract}
This paper proposed a layered fuzzy model of facial expression generation, in which the layers of physiological model at low level, emotional model at middle level and social rules at high level determine the fuzzy facial expression generation. In the layered fuzzy facial expression generation system, facial expressions of 26 emotions can be fuzzily generated, as well as social expressions and instinctive expressions. Experiment results indicated that the model of layered fuzzy facial expression generation works well in displaying lifelike facial expressions.
\end{abstract}

Keywords: Facial expression generation, fuzzy theory, layered fuzzy model.

\section{Introduction}

Facial expression takes an important role in human's daily life, and it has been widely researched in psychology, sociology, cognitive science, biology, neuroscience and so on. In the "Emotion View", kinds of facial expressions of emotions were studied by Darwin ${ }^{[1]}$ and facial expressions of 6 basic emotions were pointed by Ekman and Friesen ${ }^{[2]}$ to be universally recognized. On the contrary, the "Behavioral Ecology View" treats facial displays as expressions as social signals of intent ${ }^{[3]}$. Recently, facial expressions have been considered as emotional activators and regulators ${ }^{[3]}$.

Along with the rapid development of computer science, human-computer interaction and related fields, the generation of facial expressions in computer has been actively researched. In the Improvisational Animation system ${ }^{[4]}$, facial expressions such as angry, daydreaming, disgusted, distrustful, fiendish, haughty etc. were realized by relate lower level layer facial movements to higher level moods and intentions. In the fuzzy rule-based system ${ }^{[5]}$, an animated agent's representations of 7 single expressions and blending expressions of 6 basic emotions of were mapped onto muscle contraction values. In the CharToon system ${ }^{[6]}$, facial expressions were obtained by interpolation between the 7 known expressions (neutral, sadness, happiness, anger, fear, disgust, and surprise) positioned on the emotion disc. In the visual-speech animation system ${ }^{[7]}$, a variety of emotional expressions can be obtained by blending 6 well-characterized emotional key-expressions (including neutral, happy, sad, surprised, afraid, and angry expressions). In the intelligent agent architecture ${ }^{[8]}$, emotional intelligence was introduced into an animated character to express felt emotions and expressed emotions. 
However there are some limits in the researches above. The first limit is that facial expressions are mostly constrained in the 6 basic emotions: happiness, sadness, surprise, anger, disgust and fear. Even blends of the 6 basic emotional expressions do not often appear in our daily life and can't be related to distinct emotional categories. As human can display facial expressions of many emotions, why computers do not? The second limit is that facial expressions are merely related to emotions. However, emotion is not the only source of facial expressions. For example, one winks to give a hint to someone or because he is too tired. Lifelike facial expression generation should be more complicated. The third limit is the monotone of facial expressions. Most works correlate one model of facial expression to one emotion, just with different intensities, but human don't. For example, human display kinds of facial expressions to express happiness, such as smile with mouth open or closed, symmetrically or asymmetrically, even with head wobbled.

To solve the above limits in facial expression generation, this paper proposed a model of layered fuzzy facial expression generation, in which the layers about social rules, emotions and physiological conditions were considered and fuzzy theory was used to generate mutative and rich facial expressions.

This paper is organized as follows. In the next section, a novel model of layered fuzzy facial expression generation was proposed. Then, the layered fuzzy facial expression generation (LFFEG) system was realized. Before the conclusion, the evaluation of the LFFEG system was given.

\section{Model of Layered Fuzzy Facial Expression Generation}

\subsection{Fuzzy Facial Expression}

Fuzzy is the ubiquitous character of human mind and objects. Human displays facial expressions fuzzily in real life. As human face is capable of as many as 250,000 expressions, it is impossible to relate an expression to an exact emotion or intention. Also, as human's emotions are rich and mutative, a single facial expression can not express an emotion exactly. For example, more than 60 kinds of facial expressions about anger have been found ${ }^{[9]}$.

\subsection{What Influence Facial Expression}

There are many factors that influence mapping emotional state to facial expression, such as the intensity and type of emotion, how emotional state elicited, social display rules and whether the person was encouraged to express or suppress emotion ${ }^{[9]}$. Also, the speed, amount and duration of facial expression are different from individuals ${ }^{[3]}$.

However, emotions are not the only source of facial expressions ${ }^{[10]}$. The sources of facial expressions include mental states (felt emotions, conviction and cogitation), verbal communication (illustrators, listener responses and regulators), non-verbal communication (unfelt emotions, emblems and social winks), physiological activities (manipulators, pain and tiredness). 


\subsection{Various Mechanisms for Generation}

Human's emotion is generated not only by cognitive illation but also by non-cognitive factors. Sloman proposed a structure assuming that human's brain has three layered structure: reactive layer, deliberative layer and self-monitoring layer ${ }^{[11]}$. Reactive layer was used to generate fast and first emotion. Deliberative layer was related to second emotion generated by cognition. The self-monitoring layer was the layer where self concept worked weightily.

As human's facial expression is innate and social, influenced by emotional, physiological and social factors, facial expression generation based on various mechanisms is reasonable.

\subsection{Layered Fuzzy Facial Expression Generation}

Based on the fuzzy character of facial expression, kinds of factors that influence facial expression and the various-mechanisms-based generation, the model of layered fuzzy facial expression generation was proposed. As seen in fig.1, the layers of physiological model at low level, emotional model at middle level and social rules at high level determine the fuzzy facial expression generation.

Emotion is considered the primary factor for facial expression generation in human computer interaction. Once emotion is determined by the motional model, emotional facial expression can be fuzzily generated. The physiological model simulates the physiological states such as pain or tiredness, which can elicit instinctive facial expression. Social rules are related to the linguistic and nonlinguistic communications, and social facial expressions are generated, working as the filter to the emotional and instinctive facial expressions.

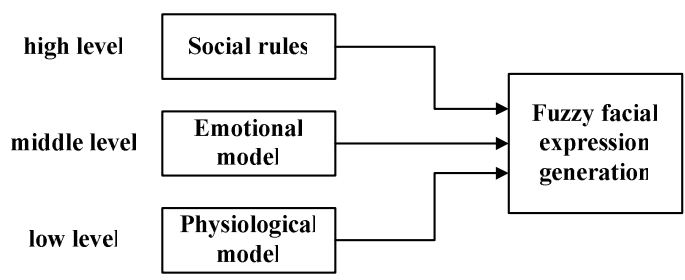

Fig. 1. Model of layered fuzzy facial expression generation

If emotion is always expressed in a changeless way, it is easy to be recognized by human or computer. However, most emotions are not mapped to a fixed form of facial expression in real life. Fuzzy facial expression generation maps one emotion to different facial expressions, making facial expressions more smart and expressive.

\subsection{Layers of the LFFEG Model}

The layer of physiological model includes the physiological variables which influence human's emotion and expression. Picard recognized that hunger or pain can influence the activation of emotional states ${ }^{[9]}$. For example, hunger can increase irritability, and pain can spur anger. Also, changes in brain blood temperature along with other 
physiological changes may lead to the feeling of excitation or depressed ${ }^{[3]}$. In the LFFEG model, the physiological variables influence the emotional expressions or lead to instinctive expressions such as grimace of pain.

The layer of emotional model includes multiple emotions based on the OCC model ${ }^{[12]}$. As the 22 emotions in OCC model are well accepted in the research of affective computing, it is reasonable to research the facial expressions to display the emotions. In the LFFEG model, multiple facial expressions can be fuzzily generated according to the emotions. For example, kinds of smile facial expressions can be elicited by the emotions such as joy, appreciation, gloating, satisfaction and happy-for, fuzzily controlled by the factors such as the intensity of the emotion, the type of the emotion and the expression personality.

The layer of social rules model includes what Ekman called social display rules and some predefined social expressions. When, where and how to express facial expressions is restricted by the social rules. A felt emotion may be masked by a fake emotion due to some display rules. In the LFFEG model, the social expressions generated by social rules can exceed the affect of the emotional model. For example, whatever a waiter felt, he should be polite to the client.

In the LFFEG model, social expression, emotional expression and instinctive expression are generated from the layers of social rules, emotional model and physiological model. The relation of them is shown in fig.2, with some cross expressions with each other. Social expressions are the facial expressions such as smile, wink and plea regardless of the emotion behind. Emotional expressions are the facial expressions elicited by kinds of emotions such as happiness, sadness and so on. Instinctive expressions are the facial expressions elicited by physiological activities, including the quick emotional expressions such as surprise, horror and other expressions such as frown, blink and gape.

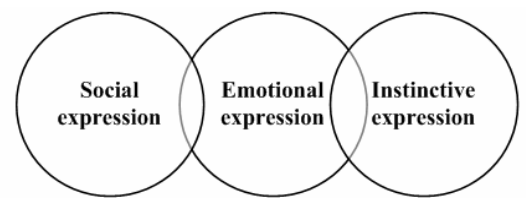

Fig. 2. The relation of facial expressions in different levels

\section{The Layered Fuzzy Facial Expression Generation System}

\subsection{System Overview}

The overview of the layered fuzzy facial expression generation system is shown in fig.3. The layers of social rules, emotional model and physiological model have respective priority, denoting the weight of the layer at a time. The module of fuzzy facial expression generation processes the output of the three layers, giving the final facial expression. Social expression is determined by the parse module from the layer of social rules. Emotional expression is determined by the fuzzy control function block from the layer of emotion model. Instinctive expression is determined by the 


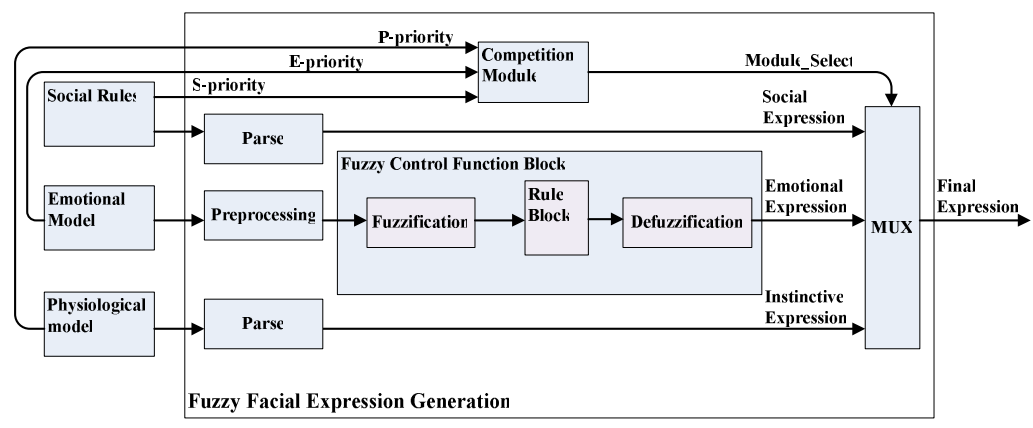

Fig. 3. Overview of the Layered Fuzzy Facial Expression Generation System

parse module from the layer of physiological model. According to the priorities of the three layers, final expression is determined from the social expression, emotional expression and instinctive expression.

The inputs of the facial expression generation are defined as followed:

1. Time: $\mathrm{t}$;

2. Emotional Parameters: $E(t)=\left\{E_{P}(t), E_{S}(t), E_{M}(t), E_{P}(t)\right\}$ is the interface of the emotional model, where $E_{P}$ : Priority, $E_{S}$ : Specific emotions, $E_{M}$ : Mood, $E_{P}$ : Expression personality;

3. Social Parameters: $S(t)=\left\{S_{P}(t), S_{E}(t), S_{R}(t)\right\}$ is the interface of the social rules, where $S_{P}$ : Priority, $S_{E}$ : Social expressions, $S_{R}$ :Social rules;

4. Physiological parameters: $P(t)=\left\{P_{P}(t), P_{V}(t)\right\}$ is the interface of the physiological model, where $P_{P}$ : Priority, $P_{V}$ : Physiological variables.

So the layered fuzzy facial expression generation function is:

$F(t)=F(E(t), S(t), P(t))$, where $F(t)$ is the fuzzy function.

\subsection{The Lingual Realization of the LFFEG}

The layered fuzzy facial expression generation is realized based on extensible markup language (XML), which provides an easy way to control the agent's behavior. Previous efforts in the XML-based languages are Human Markup Language (HML), Multimodal Presentation Markup Language (MPML), Synchronized Multichannel Integration Language (SMIL), etc. In this paper, the Layered Fuzzy Facial Expression Generation Language (LFFEGL) is developed to realize the LFFEG model.

In the LFFEGL script as seen in fig.4, the tags of "social", "emotion" and "physiological" relate to the layers of social rules, emotional model and physiological model respectively. The attribute "priority" gives the weight of the layers. Possible parameters of the LFFEGL are shown in fig.5, 5 social expressions are provided in the layer of social rules, 26 emotions are provided in the layer of emotional model and 12 physiological variables are provided in the layer of physiological model. 


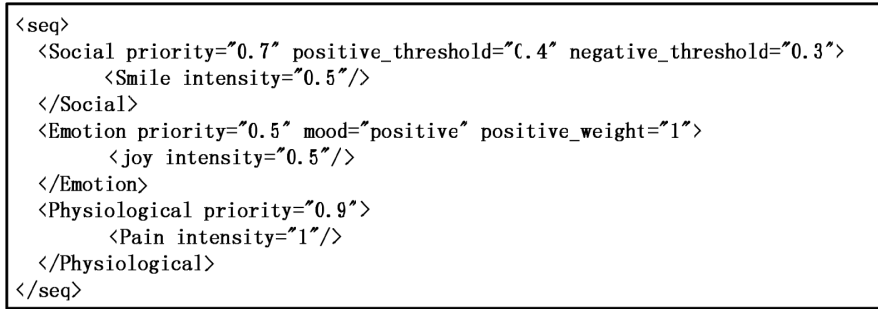

Fig. 4. Sample LFFEGL Script

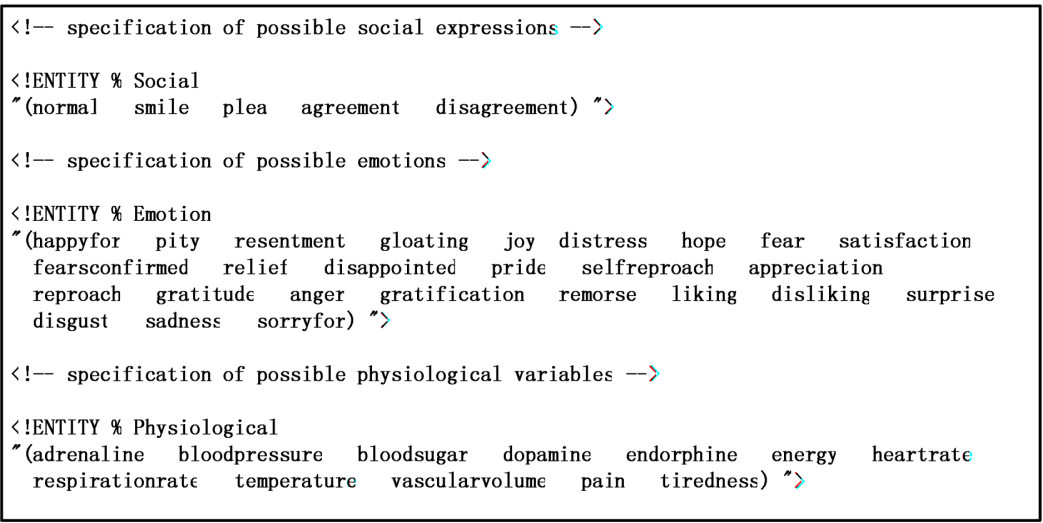

Fig. 5. Specification of the Parameters in LFFEGL

\subsection{Facial Expressions Influenced by Physiological Factors}

In the layer of physiological model, the physiological variables are chosen based on literature [13], in which emotions can arise as a result of simulated physiological changes. The physiological variables are adrenaline, blood pressure, blood sugar, dopamine, endorphine, energy, heart rate, respiration rate, temperature, vascular volume, pain and tiredness, which may influence the emotional expressions or lead to instinctive expressions. For example, high levels of endorphines can increase the expressiveness of positive emotions or decrease the expressiveness of negative emotions, or trigger a state of happiness.

\subsection{Fuzzy Emotion-Expression Mapping}

In the layer of emotional model, the arousal-valence emotional space ${ }^{[14]}$ is introduced to position the 26 emotions. Valence denotes if the emotion is positive or negative, and arousal denotes the intensity of the emotion.

The mapping of emotion to expression is many-to-many. Firstly, one emotion can be mapped to many facial expressions. For example, the fuzzy emotional facial expression of joy can be expressed as formula 1. Secondly, a predefined facial 
expression can express many emotions. For example, the emotions of joy and happyfor can be expressed as the facial expression of "SmileOpen" with different intensities.

$$
E_{\text {happyfor }}=\left\{\begin{array}{c}
a, \text { SmileClosed } \\
b, \text { SmileOpen } \\
\cdots
\end{array} \quad a, b, \cdots \in(0,1]\right.
$$

Where $a, b$ are respectively the probabilities that joy is mapped to the facial expression of "SmileClosed" or "SmileOpen".

Intensity control of predefined facial expression was realized in literature [15], however, the intensity of facial expression is not mapped directly to the intensity of the emotion. As seen in fig.6(a), the largest intensity of facial expression of "SmileClosed" may be not enough to express the largest intensity of happy-for. As seen in Fig. 6(b), moderate intensity of facial expression of "Disgust" may be enough to express the largest intensity of disliking.

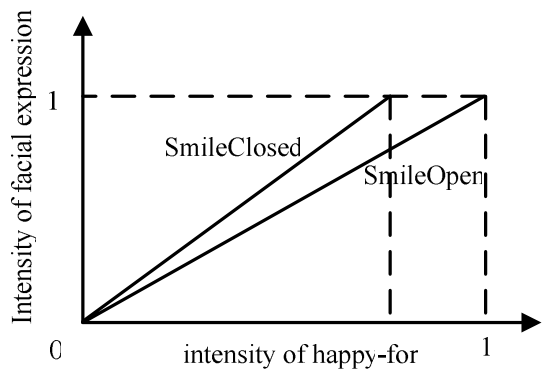

(a) happy-for

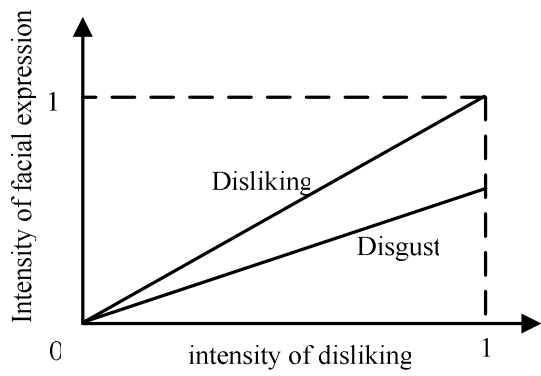

(b) disliking

Fig. 5. Mapping of intensity of emotions to intensity of facial expressions

\subsection{Expression Personality}

Facial expressiveness, like other forms of sentic modulation, is influenced by a person's innate physiology, which is related to temperament ${ }^{[9]}$. Affected by the personality, different person with the same intensity of emotion may express facial expressions with different intensities. For example, inhibited ones have lower overall facial expressiveness than uninhibited ones.

To realize the personality in facial expression, literature [16] introduced the module of Expression Personality in the signal processing structure of the robot, which is defined as a matrix of expression personality, weighting 7 emotions. Here, as there are more emotions in this system, the intensity of the emotional expression is gained by weighting the positive emotions or negative emotions with the attribute of "positive_weight" or "negative_weight", even the weight of specific emotion can be given, thus the expression personality is realized. 


\subsection{Facial Expressions Influenced by Social Rules}

Buck argued that social factors can facilitate or inhibit facial expression depending upon the nature of emotion being expressed and the expressor's personal relationship with the other ${ }^{[17]}$.

In the LFFEG system, if the layer of social rules has higher priority than layer of emotional model, the attributes "positive_threshold" and "negative_threshold" will restrict the largest intensities of the positive facial expressions and negative facial expressions respectively, acting as inhibiting facial expression. The element such as "smile" or "agreement" specifies social expression to take the place of emotional expression. The specified social expression sometimes operates as facilitating facial expression.

\section{Evaluation of the LFFEG System}

To evaluate the LFFEG system, lively facial expression animations of the character should be presented to the subjects. Xface ${ }^{[15]}$ was utilized to generate keyframes of facial expressions to display kinds of emotions. Some example keyframe facial expressions of disgust, surprise and disliking are shown in fig.7. In facial expression animation of some emotions such as pride and agreement, head movement was involved to strengthen the effect. Fuzzy facial expression animation can be generated through LFFEGL script.

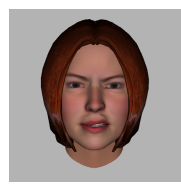

(a) disgust 1

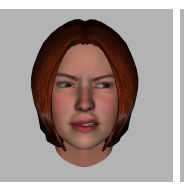

(b) disgust 2

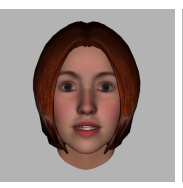

(c) surprise 1

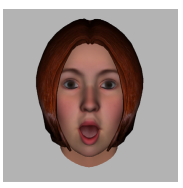

(d) surprise 2

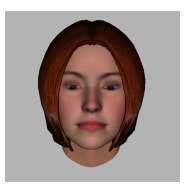

(e) disliking1

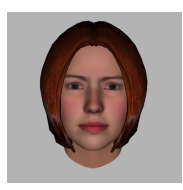

(f) disliking2

Fig. 6. Some example keyframe facial expressions

In the first experiment, 10 subjects were asked to judge 21 keyframes of facial expressions, giving what emotions each facial expression likely to present and the corresponding score of expressiveness from 1 score (low) to 5 score (high). According to the results, each emotion was related to some facial expressions and fuzzy parameters were determined in the LFFEG system.

In the following experiment, 30 scripts were written in LFFEGL to generate fuzzy facial expression animations with middle intensity. 20 subjects were asked to run each script 5 times to evaluate the effect of the fuzzily generated facial expression animations, giving the score of satisfaction from 1 score (low) to 5 score (high). The results are showed in table 1, denoting that most facial expressions acted well. For those facial expressions with low scores, better keyframes and appropriate movement of head should be taken to strengthen the expressiveness. 
Table 1. Score of satisfaction of fuzzily generated facial expressions

\begin{tabular}{l|l|l|l|l|l|l|l|l|l}
\hline F.E. & S. & F.E. & S. & F.E. & S. & F.E. & S. & F.E. & S. \\
\hline anger & 3.9 & disappointed & 2.8 & gratitude & 2.9 & sadness & 3 & agreement & 4.8 \\
fear & 3.5 & appreciation & 3.3 & happyfor & 4 & sorryfor & 2.8 & resentment & 4.1 \\
joy & 4 & fearsconfirmed & 3.4 & distress & 3.3 & relief & 3 & satisfaction & 3.7 \\
pity & 3.2 & disagreement & 3.7 & gloating & 3.5 & remorse & 3.1 & disgust & 4.8 \\
pride & 4.2 & gratification & 3.7 & disliking & 3.2 & liking & 3.5 & tiredness & 4 \\
hope & 2.8 & selfreproach & 2.5 & reproach & 3.4 & pain & 4.6 & surprise & 4.3 \\
\hline
\end{tabular}

Note: F.E.=facial expression, S.=score

Comparing with early works, more emotions are considered in our work, together with social and physiological factors. Facial expressions fuzzily generated by the layers in different levels are richer and reasonable, tally well with the character of human. The lingual realization of the LFFEG model provides an easy way to control the agent's facial expression. However, blend of emotional facial expressions is not the point in our work, as they seem amphibolous and often make people puzzled.

\section{Conclusion}

In this paper, a model of layered fuzzy facial expression generation is proposed. In the LFFEG model, the affects of the social rules, emotional model and physiological model are realized, and the generated facial expressions are lifelike, fuzzy and with personality. The lingual realization of the LFFEG makes it easy to realize and extensible. There are two primary novelties in our work: layered and fuzzy. Firstly, the factors that affect facial expression generation are considered in different layers, not only emotion but also social and physiological factors. Secondly, fuzzy facial expressions are realized to display multiple emotions, making the expression of the agent smart and rich. So the facial expression generation of the agent is more like human, making the agent intelligent in displaying facial expressions in human computer interaction.

Acknowledgments. This work is supported by the National Nature Science Foundation of China (No.60572044) and High Technology Research and Development Program of China (863 Program, No.2006AA01Z135). We also appreciate ITC-irst to provide the open source Xface toolkit for 3D facial animation.

\section{References}

1. Darwin.: The Expression of the Emotions in Man and Animals. John Murray, London (1872)

2. Ekman, P., Friesen, W.: Constants across Cultures in the Face and Emotion. J. Personality and Social Psychology 17(2), 124-129 (1971)

3. Lisetti, C.L., Schiano, D.J.: Automatic Facial Expression Interpretation: Where HumanComputer Interaction, Artificial Intelligence and Cognitive Science Intersect. Pragmatics and Cognition (Special Issue on Facial Information Processing: A Multidisciplinary Perspective), 8(1), 185-235 (2000) 
4. Perlin, K.: Layered Compositing of Facial Expression. SIGGRAPH'97 Technical Sketch, New York University Media Research Lab (1997)

5. Bui, T.D., Heylen, D., et al.: Generation of Facial Expressions from Emotion using a Fuzzy Rule based System. In: 14th Australian Joint Conf. on Artificial Intelligence, pp. 83-94 (2001)

6. Hendrix, J., Ruttkay, Zs., et al.: A Facial Repertoire for Avatars. In: Proceedings of the Workshop Interacting Agents, Enschede, The Netherlands, pp. $27-46$ (2000)

7. N.N.: An Example-based Approach to Text-driven Speech Animation with Emotional Expressions. EUROGRAPHICS 2003, 22(3) (2003)

8. Ochs, M., Niewiadomski, R., et al.: Intelligent Expressions of Emotions. In: 1st Int. Conf. on Affective Computing and Intelligent Interaction, pp. 707-714 (2005)

9. Picard, R.W.: Affective Computing. MIT Press, Cambridge, MA (1997)

10. Fasel, B., Luettin, J.: Automatic Facial Expression Analysis: A Survey. Pattern Recognition 36, 259-275 (2003)

11. Sloman, A., Croucher, M.: Why Robots will have Emotions. In: Proceedings of the Seventh Int. Conf. on AI, pp. 197-202 (1981)

12. Ortony, A., Clore, G.L., Collins, A.: The Cognitive Structure of Emotions. Cambridge University Press, Cambridge, UK (1988)

13. Canamero, D.: Modeling Motivations and Emotions as a Basis for Intelligent Behavior. In: Proceedings of the first international conference on Autonomous agents, pp. 148-155 (1997)

14. Lang, P.J.: The Emotion Probe. American Psychologist 50(5), 372-385 (1995)

15. Not, E., Balci, K., et al.: Synthetic Characters as Multichannel Interfaces. In: 7th Int. Conf. on Multimodal Interfaces, pp. 200-207 (2005)

16. Miwa, H., Takanishi, A., Takanobu, H.: Experimental Study on Robot Personality for Humanoid Head Robot. In: IEEE/RSJ Int. Conf. on Intelligent Robots and Systems, vol. 2, pp. 1183-1188 (2001)

17. Buck, R.: Social Factors in Facial Display and Communication: A Reply to Chovil and Others. J. Nonverb. Behav. 15, 155-161 (1991) 